FORSCHUNGSBERICHTE DES LANDES NORDRHEIN-WESTFALEN

Nr. 1956

Herausgegeben im Auftrage des Ministerpräsidenten Heinz Kühn von Staatssekretär Professor Dr. h. c. Dr. E. h. Leo Brandt

DK 711.454:622:63(430.1/316.2)

Dipl.-Volksw. Ludger Wierling

Institut für Siedlungs- und Wobnungswesen der Universität Münster

\title{
Landwirtschaft im städtisch-industriellen Ballungsraum
}

Untersucht am Beispiel des rheinisch-westfälischen Industriegebietes

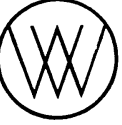

WESTDEUTSCHER VERLAG K KÖLN UND OPLADEN 1968 
ISBN 978-3-663-00743-2 ISBN 978-3-663-02656-3 (eBook)

DOI 10.1007/978-3-663-02656-3

Verlags-Nr. 011956

(c) 1968 by Westdeutscher Verlag GmbH, Köln und Opladen

Gesamtherstellung: Westdeutscher Verlag • 


\section{Vorwort}

Die fortschreitende Anpassung der Landwirtschaft an die Bedingungen der modernen Industrie- und Dienstleistungsgesellschaft läßt die Divergenz in der Bewertung der Funktionen des Agrarsektors schärfer hervortreten. Auf der einen Seite steht die Forderung nach möglichst rationeller Nahrungsmittel- und Rohstoffproduktion, auf der anderen Seite die nicht minder bedeutsame Erwartung, daß die Land- und Forstwirtschaft die gesellschaftspolitische Funktion der Erhaltung der Kulturlandschaft bestmöglich erfüllt. Diesen externen Effekten des Agrarsektors wird in der agrarpolitischen Diskussion zunehmend Beachtung geschenkt. Eine Synthese zwischen den beiden Forderungen konnte bislang noch nicht gefunden werden.

Die Problematik tritt besonders deutlich in den extremen Raumkategorien »Ballungszone « einerseits und »ländlicher Raum« andcrerscits in Erscheinung. Während sich nun zahlreiche Untersuchungen mit Funktion und Funktionswandel des ländlichen Raumes befassen, sind die spezifischen Probleme und Funktionen des Agrarsektors im Ballungsraum bisher kaum berücksichtigt worden.

In der vorliegenden Arbeit wird der Versuch unternommen, am Beispiel des rheinischwestfälischen Industriegebietes den Problemkreis "Landwirtschaft im städtischindustriellen Ballungsraum « zu durchleuchten. Dabei geht der Verfasser nicht nach ausschließlich ökonomischen Kriterien vor, sondern sieht den Agrarsektor als Teil des gesamten sozialökonomischen Systems. Diese weite Fassung des Problems ermöglicht es, ein besonderes Gewicht auf die außerökonomischen Funktionen der Landwirtschaft im Ballungsraum zu legen. Ohne Zweifel werden diese Funktionen bei den Bemühungen um die Ordnung unseres Lebensraumes an Bedeutung gewinnen.

Das Institut für Siedlungs- und Wohnungswesen hofft, mit dieser Arbeit einen Beitrag zur Klärung der angeschnittenen Fragen und zur weiteren Diskussion zu leisten. Die vorliegende Untersuchung basiert im wesentlichen auf Unterlagen und Ergebnissen eines Forschungsauftrages, den der Ministerpräsident des Landes Nordrhein-Westfalen

- Landesamt für Forschung - dem Institut für Siedlungs- und Wohnungswesen erteilt hat.

Es ist mir ein aufrichtiges Bedürfnis, auch im Namen des Verfassers an dieser Stelle allen zu danken, die die Durchführung der Untersuchung durch Hinweise und Anregungen gefördert haben. Mein besonderer Dank gilt dem Landesamt für Forschung, Düsseldorf, für die finanzielle Förderung der Arbeit und ihrer Veröffentlichung.

Münster, im Mai 1968

HANS K. SChNEIDER 


\section{Inhalt}

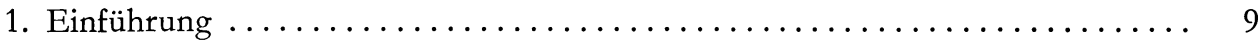

1.1 Problemstellung und Zielsetzung der Untersuchung $\ldots \ldots \ldots \ldots \ldots \ldots$

1.2 Konzeption und Methode der Untersuchung ................ 11

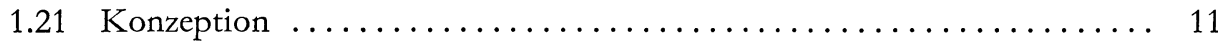

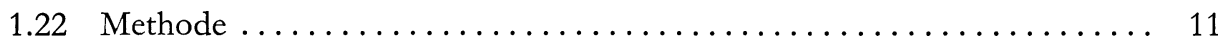

2. Die Landwirtschaft im städtisch-industriellen Ballungsraum als Problem der Wirtschafts- und Gesellschaftspolitik ...................... 13

2.T Merkmale städtisch-industrieller Ballungsräume .............. 13

2.2 Die Produktionsfaktoren als Objekte intersektoralen Wettbewerbs ..... 15

2.3 Entscheidungstheoretische Grundlagen zur Lösung konkurrierender

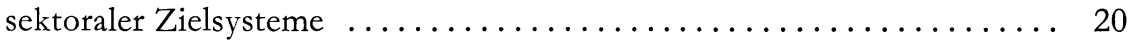

2.4 Das agrarpolitische Ziel-Mittel-System ................. 25

2.5 Diskussion des agrarpolitischen Ziel-Mittel-Systems $\ldots \ldots \ldots \ldots \ldots \ldots 27$

3. Der städtisch-industrielle Ballungsraum als Standort der landwirtschaftlichen

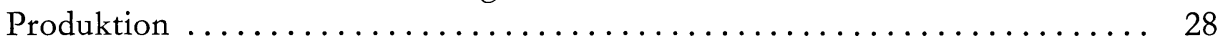

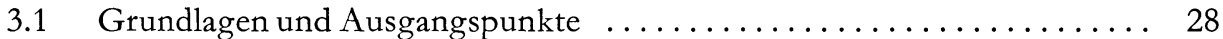

3.2 Die landwirtschaftlichen Produktionsfaktoren im Ballungsraum ...... 31

3.21 Arbeitskräfte ................................ 31

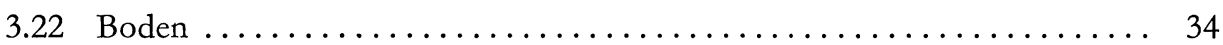

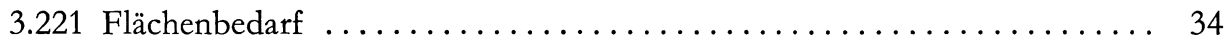

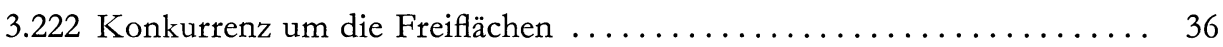

3.223 Mobilität des Bodens ............................. 37

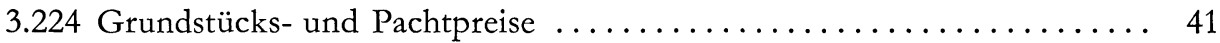

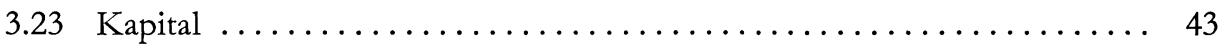

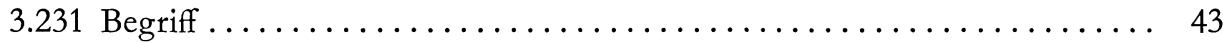

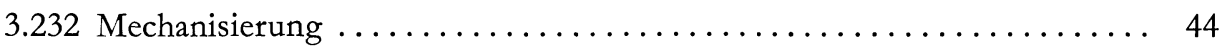

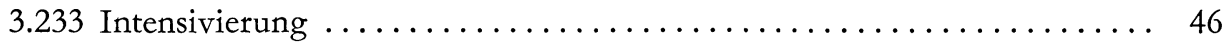

3.234 Kosten des Kapitaleinsatzes $\quad \ldots \ldots \ldots \ldots \ldots \ldots \ldots \ldots \ldots \ldots \ldots \ldots \ldots \ldots$

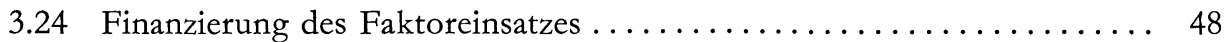

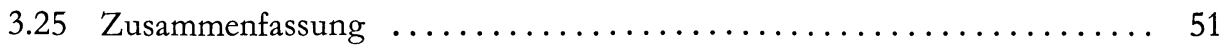

3.3 Bestimmungsgrößen für die Erlöse der Landwirtschaft im städtischindustriellen Ballungsraum ..................... 52

3.31 Determinanten der Nachfrage nach Agrarprodukten . ........... 52

3.32 Determinanten des Angebots an Agrarprodukten .............. 53

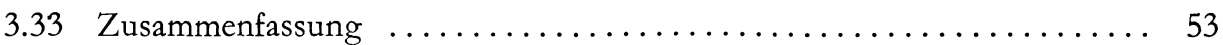

3.4 Spezifische Auswirkungen agrarpolitischer Instrumente auf die Landwirtschaft im städtisch-industriellen Ballungsraum ........... 54

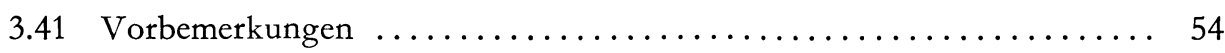


3.42 Wirkungen der Agrarmarktordnungen ................. 54

3.43 Finanzielle Staatshilfen für die Landwirtschaft $\ldots \ldots \ldots \ldots \ldots \ldots \ldots$

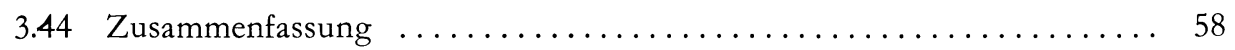

4. Strukturelle Analyse des Agrarsektors im rheinisch-westfälischen Industriegebiet 59

4.1 Charakterisierung des Untersuchungsraumes ................ 59

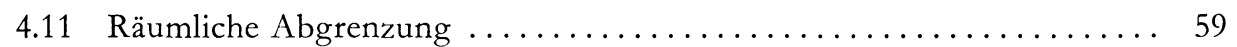

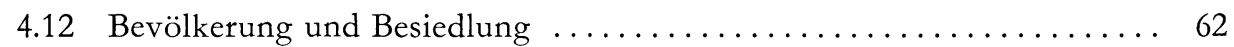

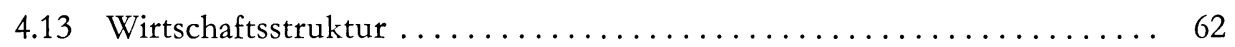

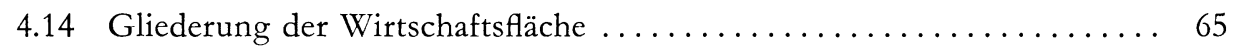

4.2 Produktionsbedingungen der Landwirtschaft $\ldots \ldots \ldots \ldots \ldots \ldots \ldots . \ldots 5$

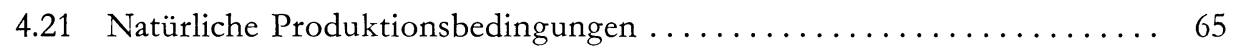

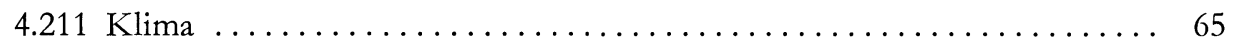

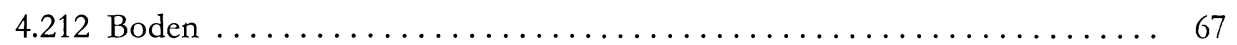

$4.22 »$ Externe Belastungen« der Landwirtschaft $\ldots \ldots \ldots \ldots \ldots \ldots \ldots \ldots 6$

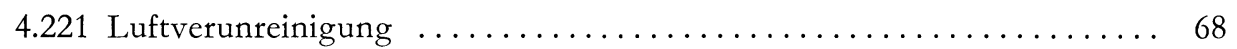

4.222 Spezielle Auswirkungen des Bergbaus $\ldots \ldots \ldots \ldots \ldots \ldots \ldots \ldots \ldots . \ldots 70$

4.23 Wirtschaftliche Produktionsbedingungen $\ldots \ldots \ldots \ldots \ldots \ldots \ldots \ldots$

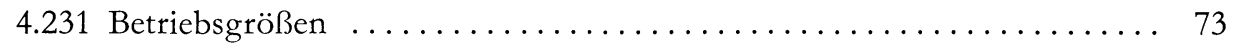

4.232 Flurverfassung und landwirtschaftliche Siedlungsstruktur ........ 78

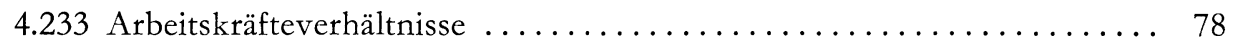

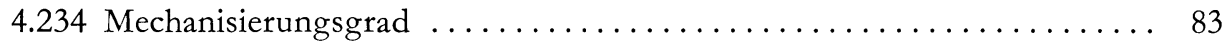

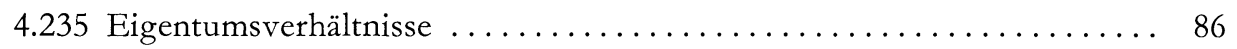

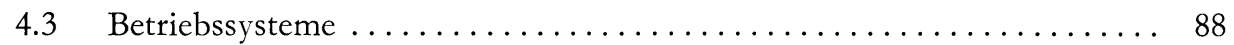

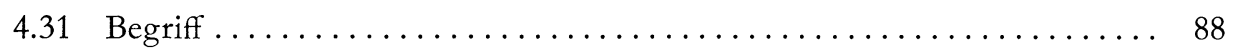

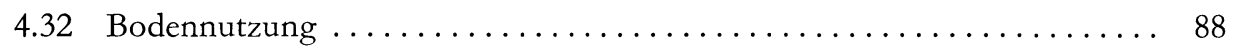

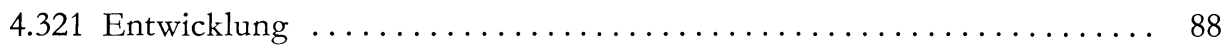

4.322 Regionale Erzeugungsschwerpunkte .................. 90

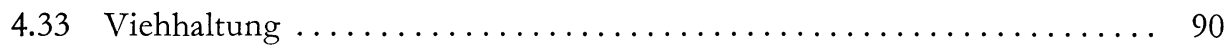

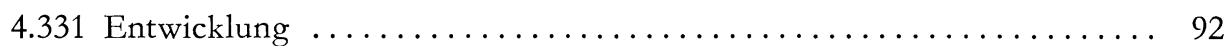

4.332 Regionale Erzeugungsschwerpunkte ................... 92

4.4 Vermarktung landwirtschaftlicher Produkte ................ 93

4.41 Vermarktungsstufen und -funktionen $\ldots \ldots \ldots \ldots \ldots \ldots \ldots \ldots \ldots \ldots$

4.42 Determinanten für die Vermarktung $\ldots \ldots \ldots \ldots \ldots \ldots \ldots \ldots \ldots . \ldots \ldots$

4.43 Produktspezifische Vermarktungsstruktur ................. 95

5. Maßnahmen zur Anpassung des Agrarsektors an die Bedingungen in der städtisch-industriellen Agglomeration » Rhein-Ruhr» ............... 98

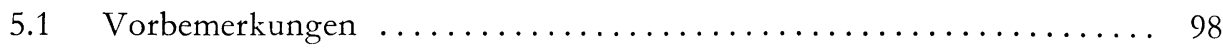

5.2 Verbesserung der Produktionsgrundlagen $\ldots \ldots \ldots \ldots \ldots \ldots \ldots \ldots . \ldots 9$

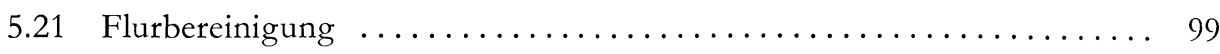

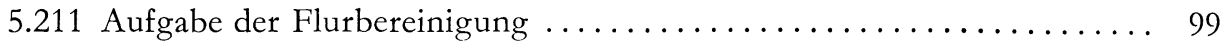

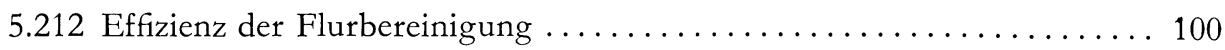


5.213 Flurbereinigung und städtisch-industrielle Expansion $\ldots \ldots \ldots \ldots \ldots 100$

5.214 Flurbereinigung und kleinbetriebliche Struktur . . . . . . . . . . 101

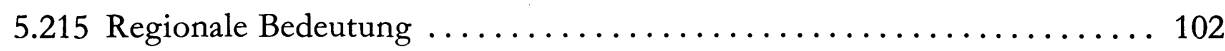

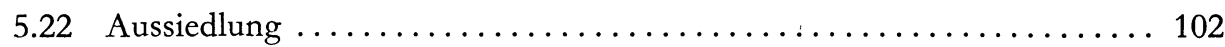

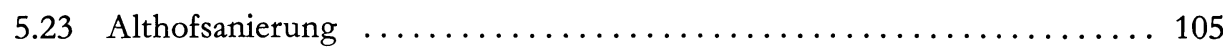

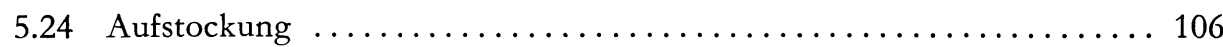

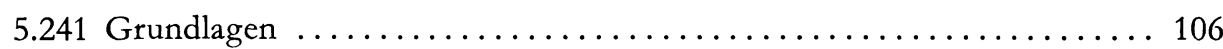

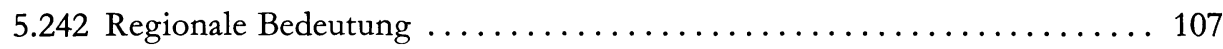

5.243 Pacht und Kauf als Aufstockungsmethoden ................ 108

5.244 Aufstockung auf Grund kurzfristiger Pachtverträge ............ 110

5.25 Zusammenfassung und Schlußfolgerungen $\ldots \ldots \ldots \ldots \ldots \ldots \ldots \ldots 112$

5.3 Betriebswirtschaftliche Anpassungsmaßnahmen . ............. 113

5.31 Betriebsvereinfachung und Spezialisierung $\ldots \ldots \ldots \ldots \ldots \ldots \ldots \ldots \ldots$

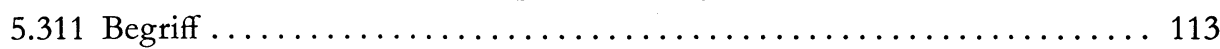

5.312 Effizienz ..................................... 113

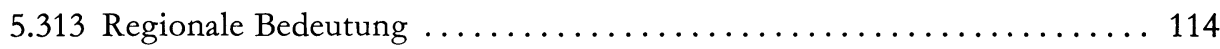

5.32 Überbetriebliche Zusammenarbeit in der Produktion ............ 119

5.321 Begriffliche Vorbemerkungen . . . . . . . . . . . . . . . . . 119

5.322 Formen und Bedeutung der überbetrieblichen Zusammenarbeit . . ... 120

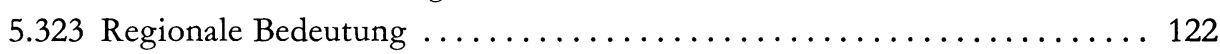

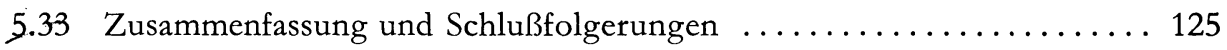

5.4 Marktwirtschaftliche Anpassung . ................... 126

5.41 Bestimmungsgründe marktwirtschaftlicher Anpassungsmaßnahmen . . . 126

5.42 Konkretisierung der Anpassungsmaßnahmen ............... 127

5.43 Zusammenfassung und Schlußfolgerungen $\ldots \ldots \ldots \ldots \ldots \ldots \ldots \ldots 1$

5.5 Einordnung des Agrarsektors in Raumordnung und Landesplanung . . 132

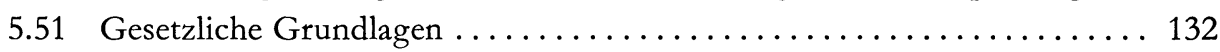

5.52 Relevanz der Planungsgesetzgebung für die Landwirtschaft . . . . . . . 133

5.521 Der Sektor Landwirtschaft im Bundesraumordnungsgesetz . . . . . . . 133

5.522 Beteiligung der Landwirtschaft an der Landesplanung nach dem nordrhein-westfälischen Landesplanungsgesetz . ............. 134

5.523 Landwirtschaftlich genutzte Räume als Planungskategorien im Landesentwicklungsprogramm für Nordrhein-Westfalen ............ 134

5.524 Die für den Agrarsektor relevanten Bestimmungen des Bundesbaugesetzes 135

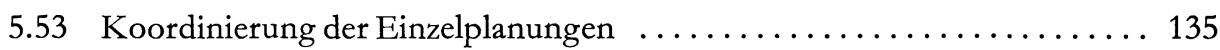

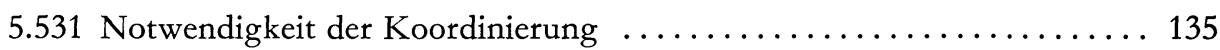

5.532 Praktische Probleme der Koordinierung . . . . . . . . . . . . 135

5.54 Die Sicherung land- und forstwirtschaftlicher Flächen als Planungsaufgabe 137

5.541 Prognose der landwirtschaftlichen Nutzfläche bis 1980 . . . . . . . . 137

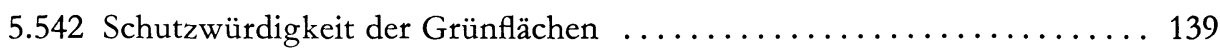

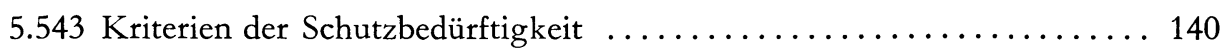

5.55 Die Problematik der landwirtschaftlichen Flächennutzung im Ballungsraum 
5.551 Der Konflikt: Erholungsraum und landwirtschaftliches Gebiet . . . . . 142

5.552 Der Konflikt: Erholungsverkehr und Landbewirtschaftung . . . . . . . 142

5.553 Immissionen und landwirtschaftliche Produktion ............... 143

5.56 Möglichkeiten zur Lösung der Konflikte . . . . . . . . . . . . . 143

5.561 Beseitigung der durch den Erholungsverkehr bedingten Belastungen ... 143

5.562 Modifizierung der Vorstellungen über den Charakter von Erholungs-

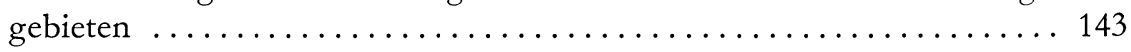

5.563 Anerkennung der Landbewirtschaftung als » öffentlicher Dienst « . . . . 144

5.564 Überführung landwirtschaftlicher Nutzflächen in die öffentliche Hand . . 145

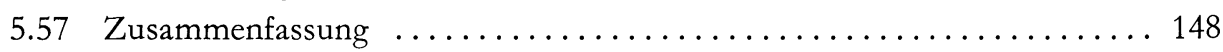

6. Zusammenfassung und abschließende Beurteilung der Untersuchungsergebnisse 148

6.1 Zusammenfassung der Untersuchungsergebnisse ............. 148

6.2 Abschließende Beurteilung der Untersuchungsergebnisse . . . . . . . 150

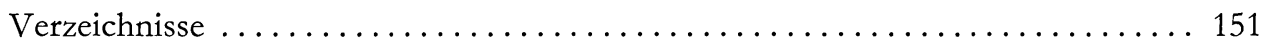

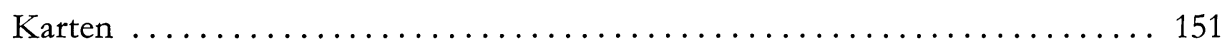

Schaubilder .................................... 151

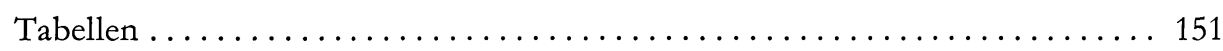

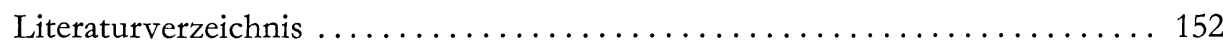

